\title{
MR-based proton density fat fraction (PDFF) of the vertebral bone marrow differentiates between patients with and without osteoporotic vertebral fractures
}

\author{
F. T. Gassert ${ }^{1}$ (D) A. Kufner ${ }^{1}$ - F. G. Gassert ${ }^{1}$ - Y. Leonhardt ${ }^{1}$ - S. Kronthaler ${ }^{1} \cdot$ B. J. Schwaiger ${ }^{1,2} \cdot$ C. Boehm $^{1}$. \\ M. R. Makowski ${ }^{1}$ ·J. S. Kirschke ${ }^{2}$-T. Baum ${ }^{2}$ - D. C. Karampinos ${ }^{1}$ - A. S. Gersing ${ }^{1}$
}

Received: 1 September 2020 / Accepted: 3 September 2021 / Published online: 18 September 2021

(c) The Author(s) 2021, corrected publication 2022

\begin{abstract}
Summary The bone marrow proton density fat fraction (PDFF) assessed with MRI enables the differentiation between osteoporotic/osteopenic patients with and without vertebral fractures. Therefore, PDFF may be a potentially useful biomarker for bone fragility assessment.

Introduction To evaluate whether magnetic resonance imaging (MRI)-based proton density fat fraction (PDFF) of vertebral bone marrow can differentiate between osteoporotic/osteopenic patients with and without vertebral fractures.

Methods Of the 52 study patients, 32 presented with vertebral fractures of the lumbar spine $(66.4 \pm 14.4$ years, $62.5 \%$ women; acute low-energy osteoporotic/osteopenic vertebral fractures, $N=25$; acute high-energy traumatic vertebral fractures, $N=7$ ). These patients were frequency matched for age and sex to patients without vertebral fractures $(N=20,69.3 \pm 10.1$ years, $70.0 \%$ women). Trabecular bone mineral density (BMD) values were derived from quantitative computed tomography. Chemical shift encoding-based water-fat MRI of the lumbar spine was performed, and PDFF maps were calculated. Associations between fracture status and PDFF were assessed using multivariable linear regression models.

Results Over all patients, mean PDFF and trabecular BMD correlated significantly $(r=-0.51, P<0.001)$. In the osteoporotic/osteopenic group, those patients with osteoporotic/osteopenic fractures had a significantly higher PDFF than those without osteoporotic fractures after adjusting for age, sex, weight, height, and trabecular BMD (adjusted mean difference [95\% confidence interval], 20.8\% [10.4\%,30.7\%]; $P<0.001$ ), although trabecular BMD values showed no significant difference between the subgroups $(P=0.63)$. For the differentiation of patients with and without vertebral fractures in the osteoporotic/osteopenic subgroup using mean PDFF, an area under the receiver operating characteristic (ROC) curve (AUC) of $0.88(P=0.006)$ was assessed. When evaluating all patients with vertebral fractures, those with high-energy traumatic fractures had a significantly lower PDFF than those with low-energy osteoporotic/osteopenic vertebral fractures $(P<0.001)$. Conclusion MR-based PDFF enables the differentiation between osteoporotic/osteopenic patients with and without vertebral fractures, suggesting the use of PDFF as a potential biomarker for bone fragility.
\end{abstract}

Keywords bone marrow $\cdot$ magnetic resonance imaging $\cdot$ osteoporosis $\cdot$ spine

Key findings

- PDFF allows the differentiation between osteoporotic/ osteopenic patients with and without vertebral fractures.

- PDFF may be a useful tool for fragility assessment in addition to the BMD.

F. T. Gassert

florian.gassert@tum.de

1 Department of Radiology, Klinikum Rechts Der Isar, School of Medicine, Technical University of Munich, Ismaningerstr. 22, 81675 Munich, Germany

\section{Abbreviations}

PDFF Proton density fat fraction

BME Bone marrow edema

BMD Bone mineral density

DXA Dual-energy x-ray absorptiometry

2 Department of Neuroradiology, Klinikum Rechts Der Isar, School of Medicine, Technical University of Munich, Munich, Germany 


$\begin{array}{ll}\text { QCT } & \text { Quantitative CT } \\ \text { BMFF } & \text { Bone marrow fat fraction } \\ \text { MRS } & \text { Magnetic resonance spectroscopy } \\ \text { MDCT } & \text { Multislice detector CT } \\ \text { STIR } & \text { Short-tau inversion recovery } \\ \text { ANOVA } & \text { Analysis of variance } \\ \text { ROC } & \text { Receiver operating characteristic } \\ \text { RMSCV } & \text { Root mean square coefficient of variation } \\ \text { AUC } & \text { Area under the ROC curve } \\ \text { QSM } & \text { Quantitative susceptibility mapping } \\ \text { HU } & \text { Hounsfield unit }\end{array}$

\section{Introduction}

Osteoporosis is a systemic disease characterized by a low bone mass and microarchitectural deterioration of bone tissue [1]. The impaired bone stability increases the risk of fractures and subsequent complications [2,3]. Therefore, a detection of the disease as early as possible and reliable assessment of osteoporosis and the evaluation of the risk of vertebral fractures in osteoporotic and osteopenic patients are necessary to prevent vertebral fractures and the following complications.

Dual-energy x-ray absorptiometry (DXA) and quantitative CT (QCT) are currently the clinical standards for assessing osteoporosis by determining the bone mineral density (BMD). Yet, QCT has previously shown to be more precise regarding BMD measurements compared to DXA [4]. For both types of BMD assessment, the patient is exposed to radiation [5]. Therefore, alternative radiation-free techniques and imaging biomarkers need to be investigated in order to evaluate their usefulness for the assessment of bone stability as well as the prediction of the fracture risk and to differentiate between the causes of fractures (e.g., low-energy osteoporotic/osteopenic vs. high-energy traumatic fractures). Consequently, the fracture risk of patients with increased bone fragility can be reduced by an adequate and timely treatment with an individualized therapeutic concept [3]. The vertebral bone encloses bone mineral matrix, adipocytes, and iron-containing hematopoietic red blood cells. It is well known that osteoporosis is associated with an increased bone marrow fat fraction (BMFF), since the differentiation of mesenchymal stem cells shifts to adipocytes rather than to osteoblasts [6-8]. Previous studies have shown that MRI-based proton density fat fraction (PDFF) measurements are a reliable tool for the assessment of vertebral bone marrow water-fat composition [9, 10]. PDFF maps allow a quantitative assessment of bone marrow fat with the benefit of avoiding radiation exposure [11, 12]. Previous studies have shown an inverse correlation between the bone marrow fat fraction and BMD $[1,13,14]$ and that PDFF is a useful tool for the differentiation between benign and malignant vertebral lesions $[15,16]$. Patsch et al. assessed the bone marrow fat content and composition in diabetic and non-diabetic women with and without prevalent fragility fractures and found a link between altered bone marrow fat composition, fragility fractures, and diabetes [17]. A further study showed that higher marrow fat was associated with prevalent vertebral fractures in men, even after adjusting for the BMD [18]. However, these previous studies were performed measuring the signal-weighted bone marrow fat fraction with single-voxel magnetic resonance spectroscopy (MRS). Using single-voxel MRS, the voxel can only be placed in one vertebral body for each acquisition. Moreover, in MRS, acquisition takes longer, and the resolution of the voxel is lower compared to chemical-shift encoding-based water-fat separation. However, MRS has the advantage of visualizing a broader biochemical spectrum than chemical-shift encoding-based water-fat separation. Therefore, both techniques are considered to be potential biomarkers for bone stability. Regis-Arnaud et al. found a significant correlation between the bone marrow fat fraction assessed using MRS and in/opposed-phase MR imaging. Moreover, in this previous study, both methods allowed the differentiation between acute and chronic benign vertebral fractures [19]. To our knowledge, it remains unknown whether spatially resolved bone marrow PDFF measurements of the entire lumbar spine, derived from six-echo 3D multi-echo gradient-echo sequences, enable the differentiation between osteoporotic/ osteopenic patients with and without vertebral fractures as well as differentiation between high-energy traumatic and low-energy osteoporotic/osteopenic fractures.

Therefore, the first aim of this study was to evaluate the diagnostic value of vertebral bone marrow PDFF measurements derived from six-echo 3D multi-echo gradient-echo sequences in osteoporotic/osteopenic patients with and without vertebral fractures. The second aim was to assess whether PDFF values are a useful tool for the differentiation between low-energy osteoporotic/osteopenic and highenergy traumatic vertebral fractures.

\section{Methods}

\section{Patient selection and study design}

Patients with a suspected vertebral fracture who underwent a CT scan at our institution as part of the clinical routine diagnostic protocol from January 2018 until July 2019 were recruited for this study. Vertebral fractures caused by an adequate high-energy trauma, that may cause fractures in patients with normal bone stability, were classified as acute high-energy traumatic vertebral fractures (e.g., patients injured in high-speed car accidents $(N=4)$, injured during a skiing accident $(N=1)$, injured during great high 
fall $(N=2))$. Vertebral fractures detected in patients with a sudden onset of severe back pain who had experienced no trauma or outside influence that may have caused any type of fracture in patients with normal bone stability were classified as low-energy osteoporotic/osteopenic fractures. Overall, 32 consecutive patients were included in this study (mean age $66.4 \pm 14.4$ years, 20 women), of which 25 patients presented with one or multiple acute low-energy osteoporotic/osteopenic vertebral fractures of the lumbar spine, and 7 patients presented with an acute high-energy traumatic fracture. Patients with vertebral fractures were frequency matched for age and sex to patients without vertebral fractures $(N=20,69.3 \pm 10.1$ years, 14 women, Table 1$)$. Patients were only included in this study if a CT had been performed previously (less than 2 days prior to the study) as part of the clinical diagnostic work up, from which a quantitative trabecular BMD measurement could be derived [20, 21]. Exclusion criteria were contraindications for MR imaging (e.g., pacemaker or pregnancy), history of cancer, or hematologic disorders. The study was approved by the local institutional review board (Ethics Commission of the Medical Faculty, Technical University of Munich, Germany) and all patients gave written and informed consent prior to their participation in the study.

\section{Computed tomography and trabecular BMD measurements}

CT images were acquired with one dual-layer dual-energy CT (IQon Spectral CT, Philips Healthcare) and one multislice detector CT (MDCT) (Philips iCT 256, Philips Healthcare). All CT scans were obtained in the craniocaudal direction with the patient in a supine position and the following parameters, according to routine clinical protocols: collimation, $0.9 \mathrm{~mm}$; pixel spacing, $0.4 / 0.3 \mathrm{~mm}$; pitch factor, $0.8 / 0.9$; tube voltage (peak), $120 \mathrm{kV}$; and modulated tube current, 125-250 mAs. Trabecular BMD values were derived from asynchronously calibrated quantitative CT examinations in $3 \mathrm{~mm}$ reformations. Vertebrae with fractures or degenerative changes, e.g., Modic changes, were excluded from the segmentations. The trabecular bone of the entire vertebral bodies L1-L4 were segmented manually by two radiologists (F.T.G and F.G.G., both 3 years of experience in musculoskeletal imaging) in the axial plane, excluding cortical bone, and the mean HU value for each vertebra was calculated. Average HU values for each patient were then calculated by averaging the mean HU values of the vertebra L1 to L4. Fractured vertebrae were not included in HU measurements. Trabecular BMD values were then calculated from the average $\mathrm{HU}$ values with the following conversion equations, which had been developed previously with phantom calibration: $0.928 \mathrm{~g} / \mathrm{cm}^{3} \times \mathrm{HU}+4.5 \mathrm{~g} / \mathrm{cm}^{3}$ for the IQon Spectral CT and $0.855 \mathrm{~g} / \mathrm{cm}^{3} \times \mathrm{HU}+1.172 \mathrm{~g} /$ $\mathrm{cm}^{3}$ for the Philips iCT 256 [22], as described previously. Trabecular BMD cutoff values for osteoporosis/osteopenia were in line with the International Society for Clinical Densitometry (ISCD) in 2007 [23] and the American College of Radiology in 2013 [24], characterizing the groups as osteoporosis, $<80 \mathrm{mg} / \mathrm{cm}^{3}$; osteopenia, 80 to $120 \mathrm{mg} / \mathrm{cm}^{3}$; and normal, $>120 \mathrm{mg} / \mathrm{cm}^{3}$. The intrareader reproducibility was calculated by segmenting the entire vertebrae L1-L4 of all patients again after 4 weeks.

\section{Magnetic resonance imaging and PDFF assessment}

PDFF measurements at the lumbar spine were performed using a 3 T MR imaging system (Ingenia, Philips Healthcare, Release 5.4, Best, Netherlands). A six-echo 3D monopolar time-interleaved multi-echo gradientecho sequence was used [25], and the posterior coil elements integrated in the table (12-channel array) were used for the acquisition. The six echoes were acquired

Table 1 Patient characteristics

\begin{tabular}{|c|c|c|c|c|c|c|c|}
\hline \multirow[t]{2}{*}{ Characteristics } & \multicolumn{3}{|c|}{ Patients with fracture } & \multirow{2}{*}{$\begin{array}{l}\text { Patients without } \\
\text { fractures }\end{array}$} & \multicolumn{3}{|c|}{ Osteoporotic/osteopenic patients } \\
\hline & $\begin{array}{l}\text { Patients with } \\
\text { high-energy } \\
\text { fractures }\end{array}$ & $\begin{array}{l}\text { Patients with } \\
\text { low-energy } \\
\text { fractures }\end{array}$ & $\begin{array}{l}\text { All patients with } \\
\text { fractures }\end{array}$ & & $\begin{array}{l}\text { With vertebral } \\
\text { fractures }\end{array}$ & $\begin{array}{l}\text { Without verte- } \\
\text { bral fractures }\end{array}$ & All \\
\hline Total patients & 7 & 25 & 32 & 20 & 25 & 8 & 33 \\
\hline Males & 3 & 9 & 12 & 6 & 9 & 3 & 12 \\
\hline Females & 4 & 16 & 20 & 14 & 16 & 5 & 21 \\
\hline \multicolumn{8}{|l|}{ Age (years) } \\
\hline Mean & $55.85 \pm 16.90$ & $72.58 \pm 11.45$ & $66.44 \pm 14.40$ & $69.30 \pm 10.13$ & $72.58 \pm 11.45$ & $63.34 \pm 16.56$ & $70.34 \pm 12.73$ \\
\hline Median & 59 & 70 & 65 & 68 & 70 & 70 & 70 \\
\hline Range & $32-68$ & $59-90$ & $32-90$ & $42-87$ & $59-90$ & $60-70$ & $59-90$ \\
\hline Weight (kg) & $76.27 \pm 11.49$ & $67.44 \pm 18.93$ & $70.89 \pm 22.19$ & $75.38 \pm 21.43$ & $67.44 \pm 18.93$ & $76.31 \pm 17.05$ & $69.59 \pm 18.61$ \\
\hline Height $(\mathrm{cm})$ & $172.56 \pm 21.76$ & $163.34 \pm 14.59$ & $165.97 \pm 17.45$ & $168.38 \pm 17.83$ & $163.34 \pm 14.59$ & $176.04 \pm 18.24$ & $166.42 \pm 16.60$ \\
\hline
\end{tabular}


in 2 interleaves with 3 echoes per repetition time (TR) and $\mathrm{TR} / \mathrm{TE} 1 / \Delta \mathrm{TE}=8.2 / 1.3 / 1.1 \mathrm{~ms}$, field of view $(\mathrm{FOV})=626 \times 511 \times 102 \mathrm{~mm}^{3}$, voxel size $=1.8 \mathrm{~mm}^{3}$ isotropic, receiver bandwidth $=1504 \mathrm{~Hz} /$ pixel, frequency direction $=$ anterior $/$ posterior $(\mathrm{A} / \mathrm{P})$, and 1 average, scan time $=3 \mathrm{~min} 39 \mathrm{~s}$. To minimize T1-bias effects, a flip angle of $3^{\circ}$ was used [26]. For the reconstruction, an image reconstruction toolbox was used (ReconFrame, GyroTools, Switzerland). The oversampling was not removed, yielding the above extended FOV. The PDFF maps were calculated using chemical shift encoding-based water-fat separation assuming a shared $\mathrm{T} 2 *$ decay of water and fat and a multi-peak fat model specific to bone marrow [27, 28 ] and are shown with a scale from 0 to $100 \%$, indicating the percentage of fat in the respective area. Segmentations of the entire vertebral bodies L1 to L4 were performed manually by two radiologists (F.T.G. and F.G.G., 3 and 5 years of experience in musculoskeletal imaging) on the PDFF maps (Fig. 1) according to previous literature [29, 30] using the open-source software Medical Imaging Interaction Toolkit (MITK, German Cancer Research Center, Heidelberg, Germany). Due to the resolution of the PDFF maps, the radiologists were not able to exclude the basivertebral plexus from the segmentations. Cortical bone was not included in the analysis. Vertebrae with fractures or degenerative changes, e.g., Modic changes, were excluded from segmentation. The mean PDFF was calculated for each vertebra, and in a second step, the mean PDFF for each patient was calculated as the mean PDFF averaged over the vertebra L1 to L4 of each patient. Moreover, the MR imaging protocol comprised a sagittal short-tau inversion recovery (STIR) as well as a T1w spin-echo sequence according to clinical standards.

The intrareader reproducibility was calculated repeating the segmentations in all patients after 8 weeks and reanalyzing the measurements.

\section{Imaging analysis}

Two radiologists (B.J.S. and A.S.G, both 8 years of experience in musculoskeletal imaging) assessed all morphologic imaging features of the vertebral fractures: height of the anterior and posterior vertebral edge in the mid-sagittal plane and height loss according to Genant et al. [31], fracture classification according to AO/Magerl et al. [32, 33], and the differentiation of acute vs. chronic vertebral fractures according to Hedderich et al. [34]. In patients with more than one fracture, each level was evaluated separately.

In total, 47 vertebral fractures were found. By analyzing the T1-weighted and STIR sequences as well as the patient's

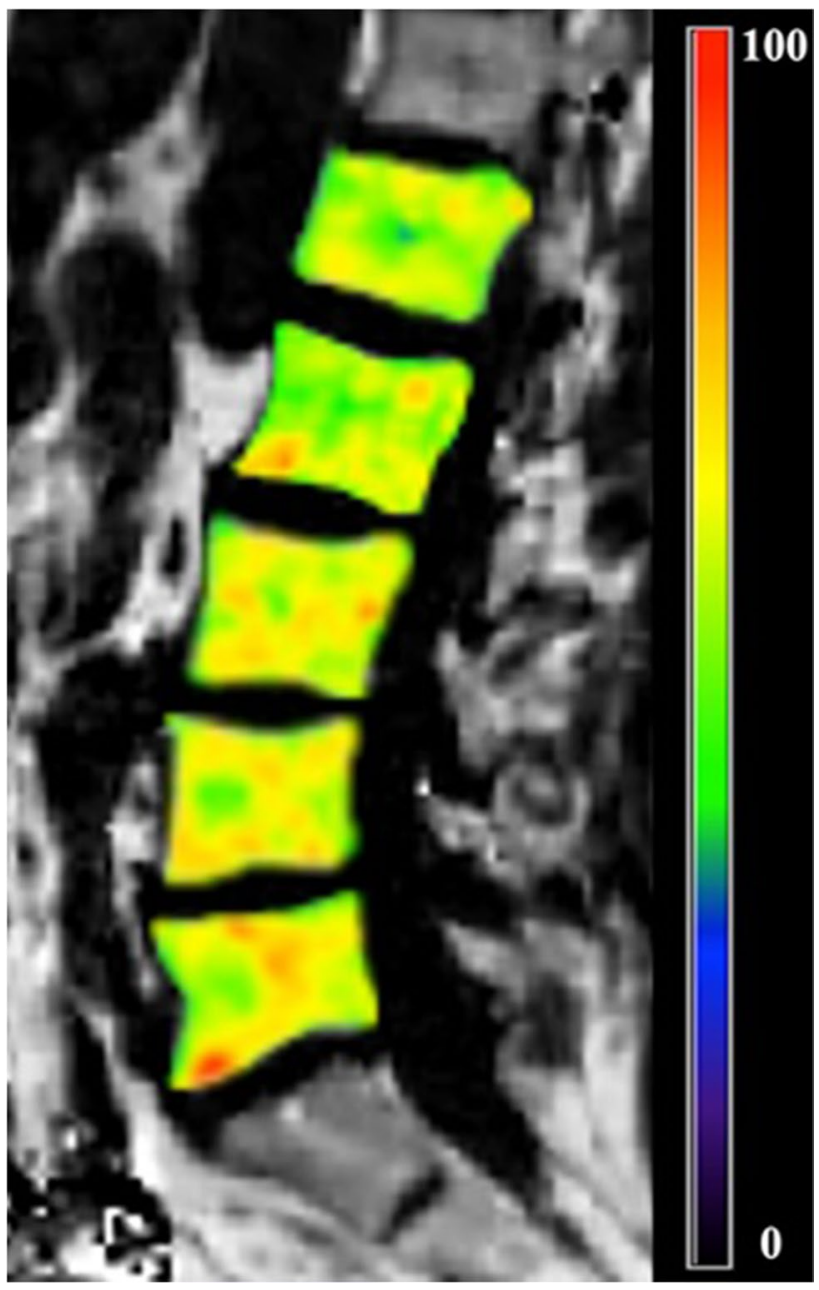

Fig. 1 Proton density fat fraction (PDFF) map obtained from the six-echo 3D monopolar time-interleaved multi-echo gradient-echo sequence with manually segmented ROIs in the vertebral bodies L1 to L5 (color-coded)

clinical history, the radiologists ensured that none of the vertebral fractures were malignant.

\section{Statistical analysis}

Statistical analysis was performed using SPSS version 25 software (IBM, New York, USA) using a two-sided 0.05 level of significance. The Kolmogorov-Smirnov analysis exhibited no significant difference from a normal distribution for both PDFF and trabecular BMD values $(P>0.1)$ in all analyzed subgroups. Pearson correlation coefficient was used to assess the correlations between PDFF, trabecular $\mathrm{BMD}$, as well as demographic data of the patients. Differences in sex between subgroups (no fracture, high-energy traumatic fracture, low-energy osteoporotic fracture) were analyzed using a Chi-square test. Differences in PDFF and trabecular BMD between subgroups were analyzed using 
a $t$-test. Differences in PDFF between patients with and without vertebral fractures in the osteoporotic/osteopenic group as well as differences in PDFF between patients with low-energy osteoporotic/osteopenic vertebral fracture and high-energy traumatic fracture were subsequently calculated using a multivariable linear regression model, adjusting for age, sex, weight, height, and trabecular BMD. Moreover, due to the number of parameters, the data were split into primary data and exploratory data before the analyses were performed. The assessment of the differences between osteoporotic/osteopenic patients with and without vertebral fracture was considered to be the primary outcome variables. The assessment of the differences between low-energy vertebral fractures and high-energy traumatic vertebral fractures were considered to be the exploratory outcome variables. Receiver operating characteristic (ROC) curve analyses were performed in order to assess cutoff values as well as the sensitivity and specificity of PDFF values for the differentiation between patients with and without fractures, using a logistic regression model for the combined analysis of PDFF and BMD. Intra-rater and inter-rater reproducibility for PDFF and trabecular BMD values were assessed by calculating the intraclass correlation coefficient and the root mean square coefficient of variation (RMSCV) of the differences between the respective measurements.

\section{Results}

The average age of the patients in this study was $67.54 \pm 12.90$ years (Table 1). Between the frequencymatched patients with and without fractures, no significant differences were found regarding age (patients with fractures, $66.44 \pm 14.40$ years versus patients without fractures, $69.30 \pm 10.13 ; P=0.44$ ) and sex (patients with fractures, 20 females $(62.50 \%)$ versus patients without fractures, 14 females $(70.00 \%) ; P=0.58)$. For those two groups, height and weight also did not differ significantly $(P>0.05$ for both). Moreover, there was no significant differences found in patient sex, height, and weight between the group with low-energy osteoporotic/osteopenic fractures and the group with osteoporosis/osteopenia without a fracture $(P>0.05$ for both). Also, no significant difference was found in patient sex, height and weight between the group with low-energy osteoporotic/osteopenic fracture and high-energy traumatic fracture $(P>0.05$ for all). Furthermore, no significant difference was found in patient age between the low-energy osteoporotic and high-energy traumatic fracture group $(P>0.05)$. The time between the trauma (high-energy vertebral fractures) or symptom onset (low-energy vertebral fractures) and the analyzed CT scan was $13.1 \pm 21.6$ days, while it was $14.0 \pm 23.9$ days between trauma or symptom onset and the MRI scan.
Overall, $63.46 \%$ of all patients were classified as osteoporotic/osteopenic patients $\left(<120 \mathrm{~g} / \mathrm{cm}^{3}\right)$. In the group without fractures, $40.00 \%$ of the patients were classified as osteoporotic/osteopenic patients $(n=8)$, while in the group with vertebral fractures, $78.13 \%$ were classified as osteoporotic/ osteopenic $(n=25)$.

Over all patients, a significant inverse correlation was found between the mean PDFF and trabecular BMD $(r=-0.51, P<0.001)$, suggesting a high PDFF in patients with low trabecular BMD. When correlating age and mean PDFF, a significant correlation was found $(r=0.56$, $P<0.001$ ), suggesting higher PDFF in older patients. There was an inverse correlation found between the trabecular BMD and age $(r=-0.45, P=0.006)$, suggesting a lower trabecular BMD in older patients. The latter correlations appeared to differ slightly between men and women (PDFF vs. age, men, $r=0.57, P<0.001$; women, $r=0.41, P=0.04$; trabecular BMD vs. age, men, $r=-0.69, P<0.001$; women, $r=-0.39, P=0.03)$. Height and weight did not show significant correlations with the PDFF and trabecular BMD $(P>0.05)$.

In a subgroup analysis including only subjects with osteoporosis/osteopenia, the mean PDFF was significantly higher in patients with vertebral fractures compared to those without vertebral fractures $(61.1 \pm 10.1 \%$ vs. $39.2 \pm 11.2 \%$; $P<0.001$; Fig. 2). Multivariable linear regression analyses showed that this effect remained significant after adjusting for age, sex, weight, height, and BMD (adjusted mean difference [95\% confidence interval], 20.8\% [10.4\%, 30.7\%]; $P<0.001$ ), suggesting higher mean PDFF in patients with osteoporotic/osteopenic vertebral fractures compared to osteoporotic/osteopenic patients without vertebral fracture (Fig. 3). This result also remained significant after adjusting the multivariable linear regression model for the CT scanner with which the CT images were obtained from which the BMD values were assessed $(P<0.001)$. On the other hand, the respective trabecular BMD values of osteoporotic/osteopenic patients without fractures (mean: $78.5 \pm 24.1 \mathrm{~g} / \mathrm{cm}^{3}$ ) showed no significant difference compared to the trabecular BMD values of subjects with osteoporosis/osteopenia and with fractures $\left(73.3 \pm 17.1 \mathrm{~g} / \mathrm{cm}^{3} ; P=0.63\right)$. For the differentiation of patients with and without vertebral fractures in the osteoporotic/osteopenic subgroup using the PDFF, an area under the ROC curve (AUC) of $0.88(P=0.007)$ was assessed in the ROC curve analysis (Fig. 4). The Youden index showed an optimal cutoff at $44.9 \%$ (sensitivity, 0.94 ; specificity, $0.83 ; J=0.77$ ).

The AUC for the combination of the mean PDFF and the trabecular BMD values was $0.90(P=0.002)$. The respective Youden index was $J=0.81$ with an optimal PDFF adjusted for BMD cutoff at $44.7 \%$ (sensitivity, 0.96 ; specificity, 0.85 ). The intrareader (ICC, 0.997 [95\% CI, 0.996-0.999]) and interreader (ICC, 0.997 [95\% CI, 0.996-0.999]) agreement 


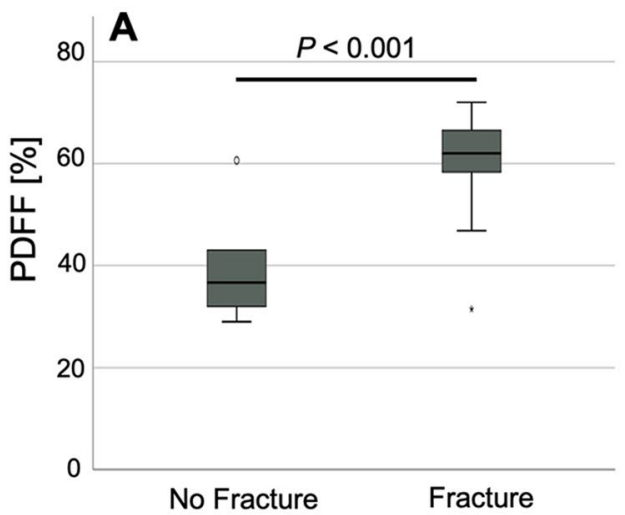

Fig. 2 A Proton density fat fraction (PDFF) of osteoporotic/osteopenic patients with $(n=25)$ and without vertebral fractures $(n=8)$. The PDFF in osteoporotic/osteopenic patients was significantly higher for those with fractures compared to those without fractures, when calculating the difference between the two groups using a multivariable linear regression analysis adjusting for age, sex, weight,

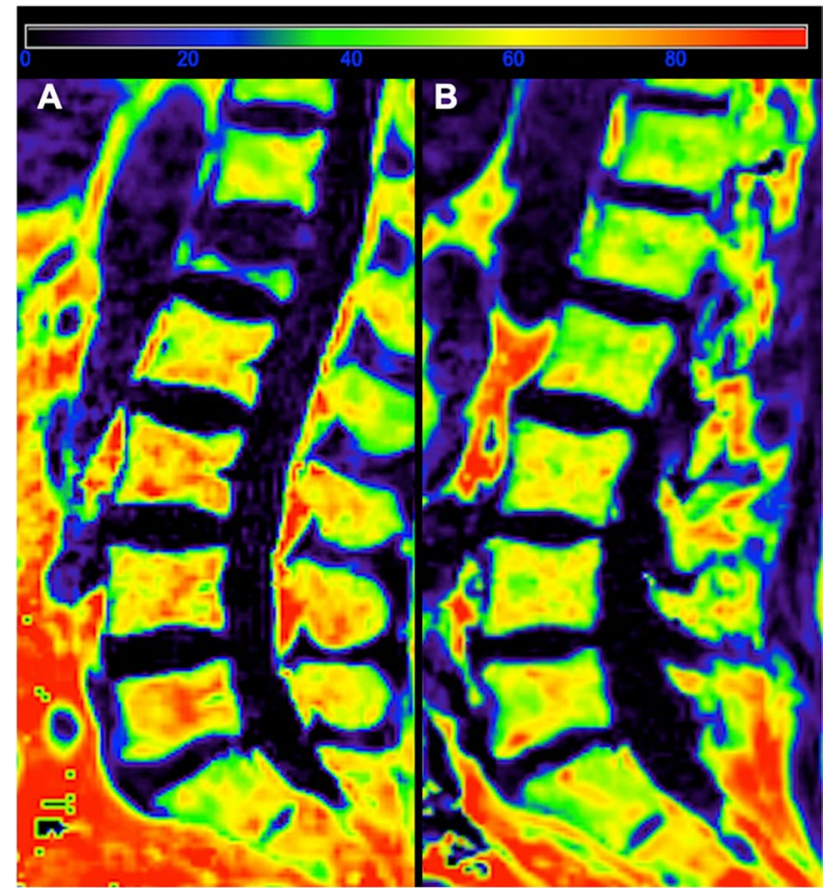

Fig. 3 Proton density fat fraction (PDFF) map (\%) of a 73-year-old female patient A with an osteoporotic vertebral fracture in L1 as well as a high PDFF in the lumbar region (red and orange regions). PDFF map of an 80-year-old female patient $\mathbf{B}$ without vertebral fracture and a low PDFF in the lumbar region (green and yellow regions)

for trabecular BMD as well as the intrareader and interreader agreement for PDFF (ICC for both, 0.976 [95\% CI, 0.959-0.999]) measurements were excellent. Intrarater reproducibility, calculated with the RMSCV, was $0.5 \%$ for the BMD analysis and $0.8 \%$ for the mean PDFF

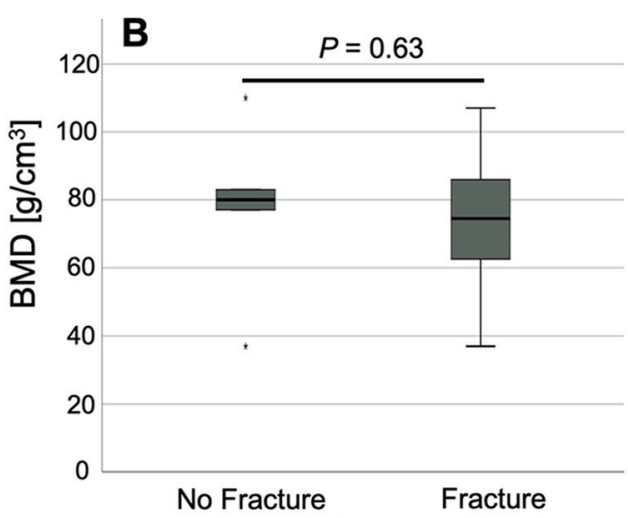

height, and trabecular BMD (adjusted mean difference [95\% confidence interval], 20.8\% [10.4\%, 30.7\%]; $P<0.001)$. B The respective trabecular BMD values of osteoporotic/osteopenic patients without fractures (mean: $78.5 \pm 23.4 \mathrm{~g} / \mathrm{cm}^{3}$ ) showed no significant difference compared to the trabecular BMD values of subjects with osteoporosis/osteopenia and with fractures $\left(73.3 \pm 17.1 \mathrm{~g} / \mathrm{cm}^{3} ; P=0.63\right)$

analysis. The interreader agreement for PDFF, calculated with RMSCV, was $0.8 \%$, and the respective interreader agreement for the trabecular BMD was $0.5 \%$. In the osteoporotic/osteopenic subgroup, no significant differences were found between patients with and without fracture for height $(163.34 \pm 14.59 \mathrm{~cm}$ vs. $167.42 \pm 13.45 \mathrm{~cm} ; P=0.49)$ and weight $(67.44 \pm 18.93 \mathrm{~kg}$ vs. $70.13 \pm 12.93 \mathrm{~kg} ; P=0.68)$.

The mean PDFF of patients with high-energy traumatic fractures was significantly lower than the PDFF of patients with low-energy osteoporotic/osteopenic fractures $(44.3 \pm 15.9 \%$ vs. $60.5 \pm 10.2 \% ; P=0.007)$. It needs to be noted that when assessing the difference in mean PDFF between the groups of patients with high-energy traumatic and with low-energy osteoporotic vertebral fractures using a multivariable linear regression model adjusting for age, sex, height, weight, and trabecular BMD, this effect did not remain significant (adjusted mean difference [95\% confidence interval $],-4.1 \%[-14.1 \%, 5.9 \%] ; P=0.41)$.

\section{Discussion}

In our study, we showed that the bone marrow PDFF measurements derived from chemical shift encoding-based waterfat separation have the potential to differentiate between osteoporotic/osteopenic patients with and without vertebral fractures, suggesting that PDFF may be a useful tool for fracture risk assessment. The PDFF differences remained significant even when adjusting for age, sex, height, weight, and trabecular BMD using a multivariable linear regression model. Moreover, the mean PDFF assessed in vertebral bodies may provide radiation-free information on bone stability, additionally to BMD. 
Fig. 4 ROC Curves showing the sensitivity and specificity for the differentiation between osteoporotic/osteopenic patients with and without vertebral fractures for the PDFF only (A) and the combination of PDFF and trabecular BMD (B)
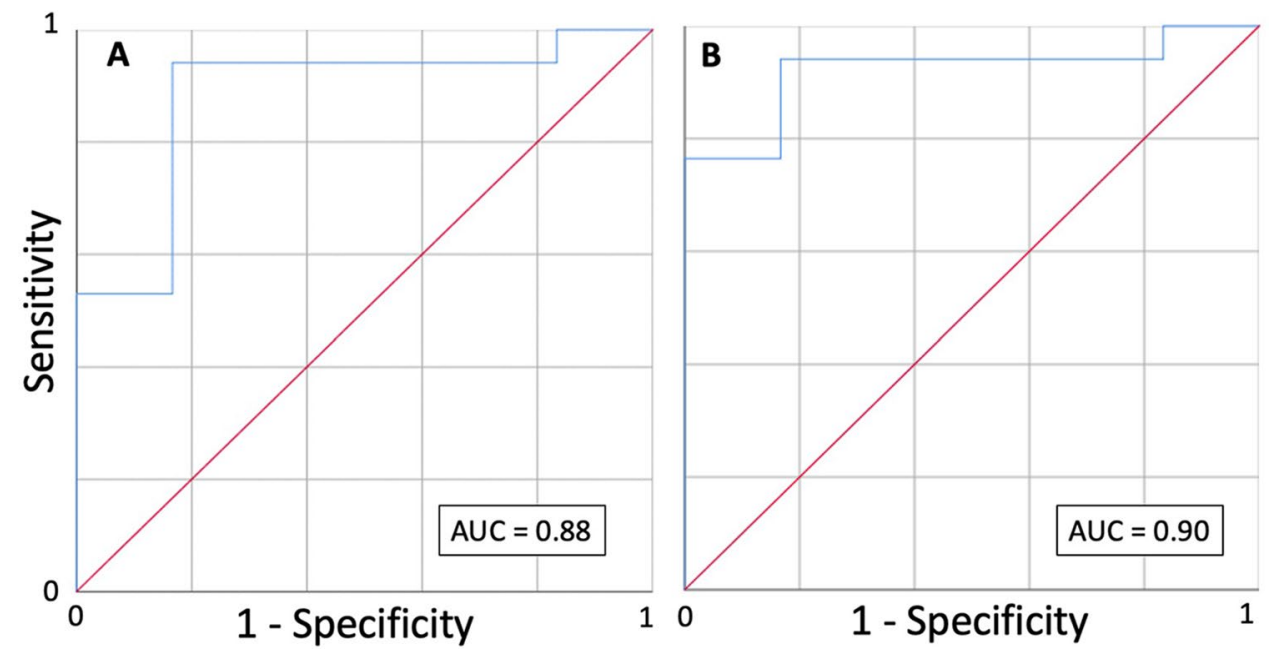

The bone marrow PDFF derived from chemical shift encoding-based water-fat separation measures a map of the density of hydrogen protons attributable to fat, normalized by the total hydrogen proton density from all mobile proton species [35]. Because of the almost equal relative proton densities of fat and water, the PDFF allows for a relative precise estimation of the fat volume fraction [36]. Therefore, PDFF has been proposed as a non-invasive tool for diagnosis of several diseases, e.g., hepatic steatosis [35, 37]. Several studies suggested that the bone marrow PDFF may also be a useful tool for the assessment of bone stability [38-43]: The gradual loss of calcium hydroxylapatite in the development of osteoporosis leads to a reduction of BMD values. The lost bone mass in the vertebral space is not simply filled with fatty bone marrow but rather includes mechanisms such as a drift in mesenchymal stem cell differentiation towards adipogenesis over osteoblastogenesis [44, 45]. A recent study analyzing data of 400 healthy individuals corrected the BMD measurements assessed for differences in bone marrow composition and investigated the relationship between BMD and PDFF [46]. Similar to our results, this previous study found a negative correlation between PDFF values and $\mathrm{BMD}$, even after correcting for differences in bone marrow adipose composition and age. A further study found that PDFF, especially in combination with simultaneous R2* measurements, may improve the prediction in differentiating healthy subjects from those with osteoporosis [1]. However, this previous study used DXA measurements for the classification of patients as healthy, osteopenic, or osteoporotic, which in that respect have shown to be less accurate compared to standard QCT BMD measurements $[47,48]$. Also, in the previous study, patients with vertebral fractures were excluded from the study, and therefore, PDFF was not evaluated for its potential to predict and/or estimate the vertebral fracture risk in osteoporotic/osteopenic patients. Another study examined quantitative susceptibility mapping (QSM) and the PDFF for the ability to assess changes in BMD, which were assessed with standard QCT [49]. Similar to our results, this previous study also found a negative correlation between PDFF and BMD measurements in the lumbar spine. Yet, this previous study demonstrated that the combination of vertebral susceptibility and fat content showed the most promising results for the MR-based detection of osteoporosis/osteopenia in postmenopausal women. However, in this previous study, the PDFF and BMD of solely postmenopausal women were assessed, and patients with vertebral fractures were excluded before the analyses were performed. Moreover, in this previous study, the rectangular ROIs were placed in the center of L1 through L3. In our study, on the other hand, the entire volume of the vertebral bodies L1 to L4 was segmented manually, in order to account for potential regional compositional differences of the vertebra and consequent regional difference of BMD and PDFF. However, it has to be noted that the negative correlation between PDFF and BMD is in part due to the fat error. This describes an underestimation of BMD measured with QCT induced by a high bone marrow fat fraction [50-53]. It has been suggested that the fat error may be less substantial when using dual-energy CT data [53]. Whether or not this could prove helpful when using opportunistic QCT acquired with a modern dual-energy CT scanner as a standard of reference for quantitative MR measurements has to be evaluated in a future dedicated analysis.

The fact that in the osteoporotic/osteopenic subgroup analysis the PDFF was higher in patients with fractures compared to those without fractures, even after adjusting for the trabecular BMD, may indicate that this effect is not just caused by the strong correlation between trabecular BMD and PDFF, but that the PDFF is an important component for the assessment of bone fragility and consequently for the assessment of the individual fracture risk of each patient. The AUC as well as the sensitivity and specificity 
improved substantially, after combining the PDFF and trabecular BMD. Therefore, an additional quantitative MRI in the diagnostic work-up of osteoporotic patients would improve the sensitivity and specificity of the fracture risk assessment substantially. However, it must be noted that age, height, and weight were different between the subgroups, yet these differences did not reach the level of significance $(P>0.05)$. The regression models were therefore adjusted for these parameters for the analyses.

Our findings show that the PDFF may provide additional information on bone fragility to the trabecular BMD. Moreover, our findings suggest that the PDFF may provide essential information for the fracture risk assessment, in addition to the opportunistic QCT. This method would therefore support the decision of the physician on the bone stability and the individual treatment selection for each patient. Even though, it is well known, that bone marrow edema (BME) in MR imaging may be helpful for differentiating between acute high-energy traumatic and chronic low-energy osteoporotic fractures [54], in this study, the presence of BME was not analyzed. However, while BME is mostly absent in later phase after osteoporotic vertebral fractures, it is regularly found in the acute phase of the osteoporotic fracture as well [55], even if the findings may be more subtle than in high-energy traumatic fractures. Further more detailed analyses regarding the difference of the edema in high-energy traumatic and low-energy osteoporotic fractures (acute and chronic) are needed in future studies.

This study has limitations. The sample size assessed in this study was fairly small. Therefore, future studies with larger study cohorts are needed to confirm our findings. Another limitation of this study is the differences in age, weight, and height between the subgroups, yet these differences did not reach the level of significance. We chose opportunistic QCT, which is not the clinical standard for BMD measurements, over DXA. Yet, standardized QCT is known to be the more accurate method for the estimation of the $\mathrm{BMD}$, and patients included in the study underwent $\mathrm{CT}$ as part of the routine clinical diagnostic work-up. While we acknowledge that standardized QCT is the standard of reference for volumetric BMD measurements, at our institution, not every patient that undergoes $\mathrm{CT}$ for a suspected vertebral fracture is examined using a standardized QCT routinely and a priori. Hence, for this study, we had to obtain opportunistic volumetric BMD measurements. However, there is evidence in the recent literature that asynchronouslycalibrated volumetric BMD measurements are accurate and reproducible [20, 55-58]. CT scans were acquired on two different scanners; however, both were standardized for trabecular BMD measurements with phantom calibration, and we therefore assumed that the trabecular BMD values are comparable without limitation. The only limitation to that is that we only assessed trabecular BMD. Another limitation of this study is that the scans were only acquired after the trauma or symptom onset and not before. A prospective study assessing PDFF and BMD before the fracture occurs is needed to assess the individual fracture risk.

In conclusion, the bone marrow PDFF is a non-invasive biomarker of bone marrow processes and allows the differentiation between osteoporotic/osteopenic patients with and without vertebral fractures. Our findings show that the PDFF may provide additional information on bone fragility to the opportunistic QCT-based trabecular BMD assessment. Therefore, PDFF may be a useful radiation-free tool for the assessment of bone fragility in addition to the BMD.

Funding Open Access funding enabled and organized by Projekt DEAL.

\section{Declarations}

\section{Conflicts of interest None.}

Open Access This article is licensed under a Creative Commons Attribution-NonCommercial 4.0 International License, which permits any non-commercial use, sharing, adaptation, distribution and reproduction in any medium or format, as long as you give appropriate credit to the original author(s) and the source, provide a link to the Creative Commons licence, and indicate if changes were made. The images or other third party material in this article are included in the article's Creative Commons licence, unless indicated otherwise in a credit line to the material. If material is not included in the article's Creative Commons licence and your intended use is not permitted by statutory regulation or exceeds the permitted use, you will need to obtain permission directly from the copyright holder. To view a copy of this licence, visit $\mathrm{http} / / /$ creativecommons.org/licenses/by-nc/4.0/.

\section{References}

1. Kuhn JP, Hernando D, Meffert PJ et al (2013) Proton-density fat fraction and simultaneous R2* estimation as an MRI tool for assessment of osteoporosis. Eur Radiol 23:3432-3439

2. Sanfelix-Gimeno G, Sanfelix-Genoves J, Hurtado I, Reig-Molla B, Peiro S (2013) Vertebral fracture risk factors in postmenopausal women over 50 in Valencia Spain. A population-based crosssectional study. Bone 52:393-399

3. Nazrun AS, Tzar MN, Mokhtar SA, Mohamed IN (2014) A systematic review of the outcomes of osteoporotic fracture patients after hospital discharge: morbidity, subsequent fractures, and mortality. Ther Clin Risk Manag 10:937-948

4. Link TM, Kazakia G (2020) Update on imaging-based measurement of bone mineral density and quality. Curr Rheumatol Rep $22: 13$

5. Link TM (2012) Osteoporosis imaging: state of the art and advanced imaging. Radiology 263:3-17

6. Fazeli PK, Horowitz MC, MacDougald OA et al (2013) Marrow fat and bone-new perspectives. J Clin Endocrinol Metab 98:935-945

7. Rosen CJ, Bouxsein ML (2006) Mechanisms of disease: is osteoporosis the obesity of bone? Nat Clin Pract Rheumatol 2:35-43 
8. Zhao LJ, Jiang H, Papasian CJ et al (2008) Correlation of obesity and osteoporosis: effect of fat mass on the determination of osteoporosis. J Bone Miner Res 23:17-29

9. Dieckmeyer M, Ruschke S, Cordes C et al (2015) The need for $\mathrm{T}(2)$ correction on MRS-based vertebral bone marrow fat quantification: implications for bone marrow fat fraction age dependence. NMR Biomed 28:432-439

10. Karampinos DC, Melkus G, Baum T, Bauer JS, Rummeny EJ, Krug R (2014) Bone marrow fat quantification in the presence of trabecular bone: initial comparison between water-fat imaging and single-voxel MRS. Magn Reson Med 71:1158-1165

11. Cordes C, Baum T, Dieckmeyer M et al (2016) MR-based assessment of bone marrow fat in osteoporosis, diabetes, and obesity. Front Endocrinol (Lausanne) 7:74

12. Karampinos DC, Ruschke S, Dieckmeyer M et al (2018) Quantitative MRI and spectroscopy of bone marrow. J Magn Reson Imaging 47:332-353

13. Li GW, Xu Z, Chen QW et al (2014) Quantitative evaluation of vertebral marrow adipose tissue in postmenopausal female using MRI chemical shift-based water-fat separation. Clin Radiol 69:254-262

14. Justesen J, Stenderup K, Ebbesen EN, Mosekilde L, Steiniche T, Kassem M (2001) Adipocyte tissue volume in bone marrow is increased with aging and in patients with osteoporosis. Biogerontology 2:165-171

15. Schmeel FC, Luetkens JA, Wagenhauser PJ et al (2018) Proton density fat fraction (PDFF) MRI for differentiation of benign and malignant vertebral lesions. Eur Radiol 28:2397-2405

16. Kim DH, Yoo HJ, Hong SH, Choi JY, Chae HD, Chung BM (2017) Differentiation of acute osteoporotic and malignant vertebral fractures by quantification of fat fraction with a Dixon MRI sequence. AJR Am J Roentgenol 209:1331-1339

17. Patsch JM, Li X, Baum T et al (2013) Bone marrow fat composition as a novel imaging biomarker in postmenopausal women with prevalent fragility fractures. J Bone Miner Res 28:1721-1728

18. Schwartz AV, Sigurdsson S, Hue TF et al (2013) Vertebral bone marrow fat associated with lower trabecular BMD and prevalent vertebral fracture in older adults. J Clin Endocrinol Metab 98:2294-2300

19. Regis-Arnaud A, Guiu B, Walker PM, Krause D, Ricolfi F, Ben Salem D (2011) Bone marrow fat quantification of osteoporotic vertebral compression fractures: comparison of multi-voxel proton MR spectroscopy and chemical-shift gradient-echo MR imaging. Acta Radiol 52:1032-1036

20. Roski F, Hammel J, Mei K et al (2019) Bone mineral density measurements derived from dual-layer spectral CT enable opportunistic screening for osteoporosis. Eur Radiol 29:6355-6363

21. Loffler MT, Sollmann N, Mei K et al (2020) X-ray-based quantitative osteoporosis imaging at the spine. Osteoporos Int 31:233-250

22. Loffler MT, Jacob A, Valentinitsch A et al (2019) Improved prediction of incident vertebral fractures using opportunistic QCT compared to DXA. Eur Radiol 29:4980-4989

23. Engelke K, Adams JE, Armbrecht G et al (2008) Clinical use of quantitative computed tomography and peripheral quantitative computed tomography in the management of osteoporosis in adults: the 2007 ISCD Official Positions. J Clin Densitom $11: 123-162$

24. ACR (2013) ACR-SPR-SSR practice parameter for the performance of quantitative computed tomography (QCT) bone densitometry The American College of Radiology. https://www.acr. org/-/media/ACR/Files/Practice-Parameters/qct.pdf. Accessed 17 Dec 2020

25. Ruschke S, Eggers H, Kooijman H et al (2017) Correction of phase errors in quantitative water-fat imaging using a monopolar time-interleaved multi-echo gradient echo sequence. Magn Reson Med 78:984-996
26. Karampinos DC, Yu H, Shimakawa A, Link TM, Majumdar S (2011) T(1)-corrected fat quantification using chemical shiftbased water/fat separation: application to skeletal muscle. Magn Reson Med 66:1312-1326

27. Diefenbach MN, Liu C, Karampinos DC (2020) Generalized parameter estimation in multi-echo gradient-echo-based chemical species separation. Quant Imaging Med Surg 10:554-567

28. Ren J, Dimitrov I, Sherry AD, Malloy CR (2008) Composition of adipose tissue and marrow fat in humans by $1 \mathrm{H}$ NMR at 7 Tesla. J Lipid Res 49:2055-2062

29. Baum T, Rohrmeier A, Syvari J et al (2018) Anatomical variation of age-related changes in vertebral bone marrow composition using chemical shift encoding-based water-fat magnetic resonance imaging. Front Endocrinol (Lausanne) 9:141

30. Dieckmeyer M, Junker D, Ruschke S et al (2020) Vertebral bone marrow heterogeneity using texture analysis of chemical shift encoding-based MRI: variations in age, sex, and anatomical location. Front Endocrinol (Lausanne) 11:555931

31. Genant HK, Wu CY, van Kuijk C, Nevitt MC (1993) Vertebral fracture assessment using a semiquantitative technique. J Bone Miner Res 8:1137-1148

32. Magerl F, Aebi M, Gertzbein SD, Harms J, Nazarian S (1994) A comprehensive classification of thoracic and lumbar injuries. Eur Spine J 3:184-201

33. Vaccaro AR, Oner C, Kepler CK et al (2013) AOSpine thoracolumbar spine injury classification system: fracture description, neurological status, and key modifiers. Spine 38:2028-2037

34. Hedderich DM, Maegerlein C, Baum T et al (2019) Differentiation of acute/subacute versus old vertebral fractures in multislice detector computed tomography: is magnetic resonance imaging always needed? World Neurosurgery 122:e676-e683

35. Reeder SB, Sirlin CB (2010) Quantification of liver fat with magnetic resonance imaging. Magn Reson Imaging Clin N Am 18(337-357):ix

36. Reeder SB, Hu HH, Sirlin CB (2012) Proton density fat-fraction: a standardized MR-based biomarker of tissue fat concentration. J Magn Reson Imaging 36:1011-1014

37. Yokoo T, Shiehmorteza M, Hamilton G et al (2011) Estimation of hepatic proton-density fat fraction by using MR imaging at 3.0 T. Radiology 258:749-759

38. Griffith JF, Yeung DK, Antonio GE et al (2006) Vertebral marrow fat content and diffusion and perfusion indexes in women with varying bone density: MR evaluation. Radiology 241:831-838

39. Li X, Kuo D, Schafer AL et al (2011) Quantification of vertebral bone marrow fat content using 3 Tesla MR spectroscopy: reproducibility, vertebral variation, and applications in osteoporosis. J Magn Reson Imaging 33:974-979

40. Yeung DK, Griffith JF, Antonio GE, Lee FK, Woo J, Leung PC (2005) Osteoporosis is associated with increased marrow fat content and decreased marrow fat unsaturation: a proton MR spectroscopy study. J Magn Reson Imaging 22:279-285

41. Griffith JF, Yeung DK, Antonio GE et al (2005) Vertebral bone mineral density, marrow perfusion, and fat content in healthy men and men with osteoporosis: dynamic contrast-enhanced MR imaging and MR spectroscopy. Radiology 236:945-951

42. Tang GY, Lv ZW, Tang RB et al (2010) Evaluation of MR spectroscopy and diffusion-weighted MRI in detecting bone marrow changes in postmenopausal women with osteoporosis. Clin Radiol 65:377-381

43. Li G, Xu Z, Gu H et al (2017) Comparison of chemical shiftencoded water-fat MRI and MR spectroscopy in quantification of marrow fat in postmenopausal females. J Magn Reson Imaging 45:66-73

44. Dunnill MS, Anderson JA, Whitehead R (1967) Quantitative histological studies on age changes in bone. J Pathol Bacteriol 94:275-291 
45. Karampinos DC, Ruschke S, Gordijenko O et al (2015) Association of MRS-based vertebral bone marrow fat fraction with bone strength in a human in vitro model. J Osteoporos 2015:152349

46. Cheng X, Li K, Zhang Y et al (2020) The accurate relationship between spine bone density and bone marrow in humans. Bone 134:115312

47. Khoo BC, Brown K, Cann C et al (2009) Comparison of QCTderived and DXA-derived areal bone mineral density and $\mathrm{T}$ scores. Osteoporos Int 20:1539-1545

48. Adams JE (2009) Quantitative computed tomography. Eur J Radiol 71:415-424

49. Guo Y, Chen Y, Zhang X et al (2019) Magnetic susceptibility and fat content in the lumbar spine of postmenopausal women with varying bone mineral density. J Magn Reson Imaging 49:1020-1028

50. Baum T, Yap SP, Karampinos DC et al (2012) Does vertebral bone marrow fat content correlate with abdominal adipose tissue, lumbar spine bone mineral density, and blood biomarkers in women with type 2 diabetes mellitus? J Magn Reson Imaging 35:117-124

51. Bredella MA, Daley SM, Kalra MK, Brown JK, Miller KK, Torriani M (2015) Marrow adipose tissue quantification of the lumbar spine by using dual-energy CT and single-voxel (1)H MR spectroscopy: a feasibility study. Radiology 277:230-235

52. Kuiper JW, van Kuijk C, Grashuis JL, Ederveen AG, Schutte HE (1996) Accuracy and the influence of marrow fat on quantitative $\mathrm{CT}$ and dual-energy X-ray absorptiometry measurements of the femoral neck in vitro. Osteoporos Int 6:25-30
53. Laval-Jeantet AM, Roger B, Bouysee S, Bergot C, Mazess RB (1986) Influence of vertebral fat content on quantitative CT density. Radiology 159:463-466

54. Loffler MT, Jacob A, Scharr A et al (2021) Automatic opportunistic osteoporosis screening in routine $\mathrm{CT}$ : improved prediction of patients with prevalent vertebral fractures compared to DXA. Eur Radiol 31:6069-6077

55. Loffler MT, Sollmann N, Burian E et al (2020) Opportunistic osteoporosis screening reveals low bone density in patients with screw loosening after lumbar semi-rigid instrumentation: a casecontrol study. Front Endocrinol (Lausanne) 11:552719

56. Loffler MT, Jacob A, Scharr A et al (2021) Automatic opportunistic osteoporosis screening in routine $\mathrm{CT}$ : improved prediction of patients with prevalent vertebral fractures compared to DXA. Eur Radiol. https://doi.org/10.1007/s00330-020-07655-2

57. Roski F, Hammel J, Mei K et al (2021) Opportunistic osteoporosis screening: contrast-enhanced dual-layer spectral CT provides accurate measurements of vertebral bone mineral density. Eur Radiol 31:3147-3155

58. Mookiah MRK, Rohrmeier A, Dieckmeyer M et al (2018) Feasibility of opportunistic osteoporosis screening in routine contrastenhanced multi detector computed tomography (MDCT) using texture analysis. Osteoporos Int 29:825-835

Publisher's note Springer Nature remains neutral with regard to jurisdictional claims in published maps and institutional affiliations. 